\title{
Current status of Chinese herbal medicine to treat allergic rhinitis in children: from the perspective of Western medicine-a narrative review
}

\author{
Jieqiong Liang^, Qinglong Gu \\ Department of Otolaryngology, Head and Neck Surgery, Children's Hospital Affiliated to Capital Institute of Pediatrics, Beijing, China \\ Contributions: (I) Conception and design: Both authors; (II) Administrative support: Q Gu; (III) Provision of study materials or patients: J Liang; (IV) \\ Collection and assembly of data: J Liang; (V) Data analysis and interpretation: J Liang; (VI) Manuscript writing: Both authors; (VII) Final approval of \\ manuscript: Both authors. \\ Correspondence to: Qinglong Gu, MD. Department of Otolaryngology, Head and Neck Surgery, Children's Hospital Affiliated to Capital Institute of \\ Pediatrics, No. 2 Yabao Road, Chaoyang District, Beijing 100020, China. Email: gq171@163.com.
}

\begin{abstract}
Allergic rhinitis (AR) has a high prevalence and significantly affects quality of life in children. According to traditional Chinese medicine theory, AR in children can be classified as BiQiu, and the etiology lies in the lung, spleen and kidney. The internal cause is primarily related to congenital deficiencies of vital qi; dysfunction of the lungs, which will lead to inhibited nose orifices; and deficiency of the spleen and kidney, which will lead to lung qi deficiency. The external cause is cold, inadequate diet and other factors. The theoretical systems of Chinese and Western medicine represent different understandings of the life science of the human body from the different perspectives of Eastern and Western culture. Therefore, ways for these approaches to communicate with and promote one another to achieve a true combination approach is the focus of our attention. In this review, we specifically discuss the mechanisms of action of herbal monomers and compounds of Chinese herbal medicines in the treatment of AR and present some results and shortcomings of Chinese herbal medicines in combination with Western medicines in the treatment of AR. We also propose future research directions and trends to enhance combined treatment with Chinese and Western medicines.
\end{abstract}

Keywords: Children; allergic rhinitis (AR); Chinese herbal medicine

Submitted Sep 16, 2021. Accepted for publication Dec 20, 2021.

doi: $10.21037 /$ tp-21-553

View this article at: https://dx.doi.org/10.21037/tp-21-553

\section{Background}

Allergic rhinitis (AR) in children is a noninfectious inflammatory disease of the nasal mucosa that occurs after the body is exposed to allergens. It is mainly mediated by immunoglobulin $\mathrm{E}$ (IgE) and is a common allergic disease. AR has become a major pediatric respiratory inflammatory disease and has a high incidence. The prevalence of $\mathrm{AR}$ in children in China is $15.79 \%$ (95\% CI: $15.13-16.45 \%$ ), and there is a trend of different incidences in different regions (1).
Allergic symptoms have a great impact on the quality of life of children with AR (2).

At present, the diagnosis of AR in Western medicine is based on clinical symptoms, etiology, triggers and signs, combined with allergen detection. "BiQiu" originated from traditional Chinese medicine (TCM) literature, illustrating a series of symptoms similar to that of AR, such as rhinorrhea etc. According to TCM theory, the etiology of AR lies in the lung, spleen and kidney. The internal causes

\footnotetext{
$\wedge$ ORCID: 0000-0001-7840-9357.
} 
are primarily related to congenital deficiencies of vital qi; dysfunction of the lungs, which leads to inhibited nose orifices; and deficiency of spleen and kidney, which leads to lung $q i$ deficiency. The external causes are cold, inadequate diet and other factors.

The treatment of AR can be summarized as a four-in-one combination of prevention and treatment. Medicines are the main treatments in both TCM and Western medicine, and most of them are prescribed to control symptoms. Doctors aim to control symptoms and reduce complications with the use of the fewest drugs and with the mildest side effects. Therefore, in Western medicine hospitals in China often include TCM in their treatment of AR; that is to say, TCM and Western medicine are integrated. A reasonable combination of Chinese and Western medicine can help improve the efficacy of treatment and reduce the effect onset time.

TCM and Western medicine are two independent branches of the medical field due to their differences in cultural background and development. The basic theory of TCM is part of traditional Chinese culture; it arises from empirical medicine, and its medical model is based on natural philosophy. The basic theory of Western medicine comes from the modern West, arises from experimental medicine, and follows a biomedical model. It is based on research in anatomy, physiology, pathology, etiology, diagnostic and treatment technology, etc. using local and microscopic research methods (3). The theoretical systems of Chinese and Western medicine are based on different understandings of human life sciences in Eastern and Western culture from different perspectives and at different levels, but the two systems share the goal of preventing and treating disease. Therefore, although the concepts of communication between Chinese and Western medicine and the integration of Chinese and Western medicine were proposed at the beginning of the 20th century, the different theories and understandings of these two approaches lead us to focus on how to facilitate communication between the two systems, promote mutuality and achieve a true combination of Chinese and Western medicine. We present the following article in accordance with the Narrative Review reporting checklist (available at https://dx.doi. org/10.21037/tp-21-553).

\section{Status of Chinese herbal medicine use}

Chinese herbal medicine involves specific dosages and forms of quality controllable, safe and effective drugs created through pharmacodynamic and clinical studies under the guidance of TCM theory. Such drugs are approved by the national drug administration, are based on TCM prescriptions and use Chinese herbs as raw materials, in accordance with the specified production processes and quality standards. For Western medicine doctors, the use of TCM mainly involves Chinese herbal medicine. Reports show that approximately $60 \%$ of Chinese herbal medicines prescribed in general hospitals are prescribed by Western medicine doctors. The author counted the prescription of Chinese herbal medicines at a large grade A hospital in Hainan and found that $96.3 \%, 97.3 \%$ and $97.7 \%$ of the Chinese herbal medicine prescriptions were provided by Western medicine doctors in 2018, 2019 and 2020, respectively. Therefore, it is essential for Western medicine doctors to master the theory of TCM in order to reasonably prescribe Chinese herbal medicines, especially in the effort to organically combine them with Western medicine treatments.

As mentioned above, Chinese and Western medicine have two different theoretical systems. TCM treatment of AR involves syndrome differentiation and treatment and primarily aims to improve the physical fitness and clinical manifestations of patients; it focuses on overall regulation, generally starting with the lung, spleen and kidney and treating them according to the presence of deficiency and excess, cold and heat. This is difficult for Western medicine doctors to do. In view of this, in 2016, the State Council issued the Outline of the Strategic Plan on the Development of Traditional Chinese Medicine (2016-2030), which aimed to establish an ideal system for Western medicine doctors studying TCM, and the document "Encouraging the move of Western medicine doctors toward the study of traditional Chinese medicine", which requires that within 5 years, 262,800 new TCM physicians and pharmacists should be added in the country. These efforts will play an important role in regulating the use of Chinese herbal medicines among Western medicine doctors and promoting the integration of TCM and Western medicine, but at present, the number of people with a complete understanding of TCM remains very small. Most Western medicine doctors prescribe Chinese herbal medicines mostly on the basis of their instructions and a shallow understanding of TCM (the state permits non-TCM practitioners who have completed some coursework in TCM during their education to prescribe Chinese herbal medicines).

Although the increasingly widespread use of Chinese herbal medicines has played an important role in human health, it has been accompanied with an increase in reports of safety issues (4). Studies have shown that Chinese herbal 
medicines rank first among the drugs associated with druginduced liver injury, surpassing even anti-tuberculosis and anti-tumor preparations; these side effects seriously endanger health of patients and significantly increasing their economic burden.

At present, the irrational use of Chinese herbal medicines has attracted attention from multiple directions (5). There are six main phenomena associated with the irrational use or abuse of Chinese herbal medicines in clinical practice: (I) poor understanding of the composition of the prescribed Chinese herbal medicines that is limited to knowledge of only some of their main components rather than the entire compound; (II) little understanding of the indications for Chinese herbal medicines, knowing only some main symptoms and not understanding the key points of adaptation; (III) lack of education and training on TCM and prescribing medications based solely on the drug name or instructions for the main treatment of the disease; (IV) using Chinese herbal medicines from a purely Western medical perspective and basing their selection on impressions of theories, such as clearing away heat and toxic substances, activating blood circulation and vasodilation; (V) blindly and arbitrarily expanding the scope of medications; (VI) using Chinese herbal medicines without differentiating among syndromes, without the ability to differentiate among syndromes or while operating completely outside of the theoretical system of TCM.

Although there are situations in which Chinese herbal medicines are used irrationally, specifically at hospitals, some departments are also applying different methods for promoting the rational use of drugs in clinical practice (6-8), such as reviewing prescriptions for Chinese herbal medicines, reminding practitioners to be vigilant in their use of TCMs, and standardizing the guidelines for the use of Chinese herbal medicines.

\section{Treatment of AR with Chinese herbal medicine}

Modern pharmacological studies have shown that TCM can exert multiple effects in the treatment of $A R$, and randomized, double-blind, placebo-controlled clinical trials and systematic reviews have shown that Chinese herbal medicine is effective in improving the nasal symptoms of seasonal and perennial AR.

\section{TCM etiology of AR}

In Plain Conversation (Suwen) Pulse Solution (Maijiepian)
AR is officially named BiQiu. Yifangbian Nandacheng has stated that "The nose extends to the lung and belongs to the spleen". The lungs open orifices in the nose and control $q i$ for respiration. Lung $q i$ deficiency is unfavorable for the nasal orifices. The spleen is the acquired foundation and the biochemical source of $q i$ and blood. Spiritual Pivot (Lingshu) Ben Shenpian believes that "lung qi deficiency leads to nasal congestion". Plain Conversation Meridians Theory suggests that drinking water into the stomach transports essence and $q i$ upward into the spleen, which dissipates $q i$ and essence and returns it to the lung, regulates the water pathways and progresses down to the bladder. Water and fluid metabolism is completed through the cooperation of the meridians and viscera of the liver, heart, spleen, lung and kidney. The changes in cold and heat during the four seasons cause changes in yin and yang in the five internal organs, and appropriate adjustments should be made. Zhang Zhongjing described the disease as "stuffy nose" and believed that the accumulation of coldness prevents lung $q i$ to disperse, and the undistributed fluid and essence lead to the rhinorrhea. Therefore, TCM believes that the pathogenesis of AR is based on a combination of internal and external factors in the human body, with internal factors as the main cause and external factors as the symptoms. AR is mostly caused by deficiency of the viscera, an unstable body surface, the entry of wind and cold through the nasal orifices as a result of deficiency in the lung $q i$, the accumulation of water and fluid, congestion of the nasal orifices and the balance between yin and yang in the nose orifices.

\section{TCM syndrome differentiation in AR}

TCM uses the syndrome differentiation of viscera as the standard for AR and clarifies the clinical disease as one of four types (9): (I) lung meridian heat syndrome and its common clinical symptoms: runny and itchy nose, nasal congestion and sneezing and swelling of the nasal mucosa accompanied by coughing, itchy throat and a red tongue covered with white fur. (II) Lung $q i$ deficiency and cold syndrome, with signs of severe nasal congestion, continuous aggravation of the condition in cold weather, shortness of breath, low voice and pallor. (III) Spleen $q i$ deficiency type, characterized by sneezing and an itchy nose, symptoms that worsen after exertion, abdominal distension and loose stools, white coating on the tongue and other symptoms. (IV) Kidney yang deficiency type, characterized by severe itchy nose and sneezing accompanied by low back and knee cold pain symptoms, nocturia and pale tongue. 


\section{Mechanism of TCM for AR treatment from the perspective of Western medicine}

In $\mathrm{AR}$, the Western medicine principles that are applied to treatment are the avoidance of exposure to allergens combined with drug therapy, immunotherapy, surgical treatment and patient education. TCM emphasizes paying equal attention to prevention, treatment and nourishment and "first preventing the disease from happening, then preventing it from progressing" by enhancing immunity, reducing susceptibility and resolving cold and heat deficiency and excess. Herbal monomers are the particular raw herbs which cannot be used directly. Compounds of Chinese herbal medicines mainly refer to traditional Chinese patent medicines, which were herbal concoctions that can be used directly.

\section{Effect and mechanism of TCM monomers on AR Angelicae daburicae}

Angelicae dahurica has pharmacological effects as an antipyretic, antispasmodic, analgesic, antibacterial, antiasthmatic and antihypertensive treatment, stimulating motor and respiratory centers, inhibiting adipocyte synthesis and photosensitivity (10), and it is widely used in the clinical treatment of rhinitis and psoriasis.

\section{Flos Magnoliae}

Flos Magnoliae, also known as Magnolia biondii Pamp., was first described in Shennong's Herbal Classic. Flos Magnoliae can treat sweating at the beginning of exogenous syndromes and is good at penetrating the nine orifices, especially the nasal orifices. Flos Magnoliae can expel wind and remove cold, and can rise to lung and stomach qi (10). Modern pharmacological studies have shown that volatile oil of Flos Magnoliae can constrict the blood vessels of the nasal mucosa, promote the absorption of mucosal secretions, protect the nasal mucosa and reduce nasal inflammation, and it has local anti-allergic, astringent, irritating and anesthetic, antibacterial and anti-inflammatory effects. In animal experiments, volatile oil of Flos Magnoliae significantly inhibited the contraction of the ileum induced by histamine (HA) and acetylcholine (Ach) in guinea pigs, strongly inhibit the allergic contraction of the sensitized ileum in guinea pigs and significantly prevent mast cell degranulation in rats, with a $33.88 \%$ protective rate against mast cells. Volatile oil of Flos Magnoliae has been shown to have an anti-allergic effect (11).

\section{Xanthii Fructus}

Xanthii Fructus has evacuating and promoting effects, can inhibit cellular immunity and can significantly inhibit the breeding of allergens (12). A 70\% ethanol extract of Xanthii Fructus can inhibit immediate mast cell-dependent anaphylaxis by stabilizing the mast cell membrane and reducing the release of allergic mediators, such as HA (13).

\section{Astragalus}

The main pharmacological components of Astragalus are polysaccharides, saponins, flavonoids, amino acids and trace elements. The excessive activation of T-helper 17 (Th17) cells is one of the key factors leading to AR. Astragalus can inhibit the inflammatory response in AR by inhibiting the differentiation of Th17 cells and the release of proinflammatory cytokines (14). The polysaccharides in Astragalus also have biological effects, such as regulating the immune response and inhibiting the inflammatory response. Studies have shown that Astragalus polysaccharides can improve the imbalance of Th1/Th2 cells in AR patients and improve the efficacy of specific immunotherapy for allergic diseases (15).

\section{Atractylodes macrocephala}

Atractylodes macrocephala is an important drug for tonifying the spleen and stomach. It contains polysaccharides, which have the effect of enhancing immune function and can reduce nasal hypersensitivity.

\section{Asarum}

Asarum has the effects of expelling wind and dispersing cold, warming the lung and promoting opening of the orifices; furthermore, it has antihistaminic and anti-allergic effects and can thereby reduce the number of allergic mediators, inhibit the efficiency of receptors and block the activation of $\mathrm{T}$ cells.

\section{Effects and mechanisms of compounds of Chinese herbal medicines in AR}

Compared with Chinese herbal medicine decoctions, Chinese patent medicines are convenient to use and can be preserved; they have high patient compliance and are easy for children to take. However, each Chinese herbal medicine has its own characteristics and effects. When administering them, it is necessary to follow the basic principle of dialectical treatment. They can be used alone as the main treatment or combined with other treatments as an auxiliary measure.

At present, there are many Chinese herbal medicines for AR on the domestic market, including Sanfeng Tongqiao Diwan, Yupingfeng granules, Biyuan Tongqiao granules, Xinqin granules, Xinyi Biyan pills, Biyankang tablets, Xiangju capsules and Biyuanshu oral liquid. The exactly efficacy will be discussed as follows (Table 1). 
Table 1 TCM compounds in treatment of AR

\begin{tabular}{|c|c|c|c|}
\hline Formula name & Herbal constituents & Indication & Function mechanism \\
\hline Xinqin granules & $\begin{array}{l}\text { Asarum (Xi Xin), Scutellaria (Huang Qin), Nepeta } \\
\text { chinensis (Jing Jie), radix Angelicae dahuricae } \\
\text { (Bai Zhi), Cinnamomum cassia (Gui Zhi), Xanthii } \\
\text { Fructus (Cang Er Zi), Acorus calamus (Shi Chang } \\
\text { Pu), Astragalus (Huang Qi), Atractylodes } \\
\text { macrocephala (Bai Zhu), and divaricate } \\
\text { saposhnikovia root (Fang Feng) }\end{array}$ & $\begin{array}{l}\text { Lung qi deficiency and } \\
\text { cold syndrome } \\
\text { the }\end{array}$ & $\begin{array}{l}\text { Reduce the nasal resistance of AR patients, } \\
\text { improve ciliary clearance ability (19) }\end{array}$ \\
\hline
\end{tabular}

TCM, traditional Chinese medicine; AR, allergic rhinitis; IgE, immunoglobulin E; IL-4, interleukin 4; TNF- $\alpha$, tumor necrosis factor $\alpha$.

\section{Sanfeng Tongqiao Diwan/Bishudiwan (BSDW)}

Sanfeng Tongqiao Diwan is mainly composed of four herbs: Scutellaria, Nepeta chinensis, Asarum, and Notopterygium incisum. Of these, Scutellaria clears lung heat; Nepeta chinensis dispels wind, relieves exterior cold, and dispels lung cold; Asarum dispels cold and dredges orifices; and Notopterygium incisum dispels cold and wind and dredges orifices. The four TCMs are combined into a prescription that can be used either cold or warm to enhance the effects of clearing heat, dispelling wind, dispersing cold and dredging orifices. It can be used for exogenous wind-cold, stagnant lung heat syndrome, symptoms of nasal congestion, runny nose, sneezing, nasal mucosal congestion and so on. Bishudiwan (BSDW) is a prescription similar with Sanfeng Tongqiao Diwan, except the composition of Notopterygium incisum. At present, two main aspects of the anti-allergic effect and mechanism of BSDW in AR are being explored: reducing the levels of $\mathrm{IgE}$ and interleukin 4 (IL-4) in the serum of $\mathrm{AR}$ models, increasing the ratio of cluster of differentiation 4 (CD4)/CD8 and the level of IL-12 (20) and neutralizing and blocking $\mathrm{IgE}$ antibodies, inhibiting antigen-antibody reactions, stabilizing mast cell membranes, preventing the release of the inflammatory mediator HA and reducing local inflammatory reactions (16).

\section{Yupingfeng granules}

Yupingfeng granules represent a classic formula for supplementing $q i$ and surface consolidation. It consists of Astragalus (also known as Astragalus mongholicus, root of Astragalus membranaceus or Astragalus mongholicus), Atractylodes macrocephala (the dried rhizome of the herb Atractylodes macrocephala), and divaricate saposhniovia root (the root of the herb divaricate saposhniovia) at a ratio of $3: 1: 1$. It is derived from the Danxi Xinfa and promotes spleen $q i$, kidney $q i$ and body protection (21). There has been 
certain evidence supporting the use of Yupingfeng granules for AR management due to its anti-inflammatory and immunoregulatory function. It is showed that Yupingfeng granules reduced the eosinophilic cells infiltration and degranulation reaction, decreased the tissue edema, reduced the immune factors including HA, IgE, IL-4 and tumor necrosis factor- $\alpha$ (TNF- $\alpha$ ) in serum (17). Some studies showed that whether Yupingfeng granules itself or combined with other antihistamines such as loratadine played a positive role in treating children AR $(22,23)$. Besides, some meta-analyses pointed out that Yupingfeng granules combined with Western medicine (nasal glucocorticoids, oral antihistamines, etc.) can significantly improve the nasal symptoms of AR patients compared with Western medicine alone, can reduce recurrence and has good safety, providing some medical evidence for the integration of traditional Chinese and Western medical treatment of AR $(24,25)$.

\section{Biyuan Tongqiao granules}

The main components of Biyuan Tongqiao granules are Flos Magnoliae, Xanthii Fructus (fried), Ephedra sinica, radix Angelicae daburicae, Menthae, Ligusticum officinale, Scutellaria, Forsythia suspensa, Chrysanthemum indicum, Trichosanthes, Rebmannia glutinosa and Salvia miltiorrbiza. They are applicable to lung $q i$ deficiency and cold syndrome. The mechanism of effect is mainly an inhibitory effect on HA-induced and serotonin-induced capillary hyperpermeability and granulomatous hyperplasia; however, they also increase surfactant protein A (SP-A) levels in the nasal mucosa and regulate the balance of Th1/Th2 and Th17 cells, thus explaining the response of AR to this treatment $(18,26,27)$. Biyuan Tongqiao granules combined with rupatadine fumarate can further improve clinical symptoms, trace elements and ventilation function in AR patients (28).

\section{Xinqin granules}

Xinqin granules are mainly composed of Asarum, Scutellaria, Nepeta chinensis, radix Angelicae daburicae, Cinnamomum cassia, Xanthii Fructus, Acorus calamus, Astragalus, Atractylodes macrocephala, and divaricate saposhnikovia root. They are used for lung $q i$ deficiency and cold syndrome. Xinqin granules combined with fluticasone propionate nasal spray can significantly reduce the nasal resistance of AR patients, improve ciliary clearance ability and have a good nasal function repair effect (19).

\section{The prospect of integrated Chinese and Western medicine}

In the treatment of $A R$, there are many studies on the combination of Chinese patent medicine and Western medicine (including intranasal corticosteroids and antihistamines, etc.), and good clinical results have been achieved. However, the combination may be more arbitrary and blind, and the specific mechanism of combination is not clear. Therefore, strengthening the cooperation and exchange of traditional Chinese and western medicine, standardizing the diagnosis and treatment of integrated traditional Chinese and Western medicine, and clarifying its mechanism of action are still the main tasks of clinical research.

\section{Recommendations}

(I) Doctors of whom whether western medicine or TCM should fully understand the mechanism of each combined drug in the integrated treatment of AR. That is to say, when Chinese patent medicines were combined with Western medicine, full attention should be paid to the interaction function, and the synergistic effects are advised, while the antagonistic effect should be avoided.

(II) Many proprietary Chinese medicines contain ingredients similar to western medicines. For example, some patent Chinese medicines contain ephedra, so more attentions should be paid to the application of western medicines containing pseudoephedrine. When recommending drugs to patients, physicians and pharmacists should better interpret the "main ingredients", "adverse reactions" and "precautions" on the instructions of proprietary Chinese medicines as far as possible.

(III) As Chinese traditional patent medicine and western medicines have their own advantages in the treatment of rhinitis, the purpose of combination is complementary and rational, it is better to avoid substance abuse because of ignorance.

\section{Acknowledgments}

Funding: None.

\section{Footnote}

Reporting Checklist: The authors have completed the Narrative Review reporting checklist. Available at https:// dx.doi.org/10.21037/tp-21-553

Conflicts of Interest: Both authors have completed the 
ICMJE uniform disclosure form (available at https://dx.doi. org/10.21037/tp-21-553). The authors have no conflicts of interest to declare.

Ethical Statement: The authors are accountable for all aspects of the work in ensuring that questions related to the accuracy or integrity of any part of the work are appropriately investigated and resolved.

Open Access Statement: This is an Open Access article distributed in accordance with the Creative Commons Attribution-NonCommercial-NoDerivs 4.0 International License (CC BY-NC-ND 4.0), which permits the noncommercial replication and distribution of the article with the strict proviso that no changes or edits are made and the original work is properly cited (including links to both the formal publication through the relevant DOI and the license). See: https://creativecommons.org/licenses/by-nc-nd/4.0/.

\section{References}

1. Ai T, Wang L, Luo R, et al. Effects of sublingual-specific immunotherapy on pulmonary function and exhaled nitric oxide levels in asthmatic children with and without allergic rhinitis. Transl Pediatr 2020;9:686-94.

2. Walker S, Khan-Wasti S, Fletcher M, et al. Seasonal allergic rhinitis is associated with a detrimental effect on examination performance in United Kingdom teenagers: case-control study. J Allergy Clin Immunol 2007;120:381-7.

3. Song YB, Meng WD, Mo CY, et al. Comparison and Connection of Traditional Chinese Medicine and Western Medicine. Journal of Traditional Chinese Medicine Management 2011;19:15-8.

4. Lin YM. Adverse reactions of Chinese patent medicine. China Journal of Pharmaceutical Economics 2016;11(3):25-6.

5. Gong Y, Gu YY, Zheng F, et al. Analysis of Influential Factors for Clinical Rational Use of Chinese Patent Medicine. China Pharmacy 2017,28:2419-21.

6. Jin R, Zhao KJ, Guo GM, et al. Expert consensus on prescription comment of Chinese traditional patent medicine for promoting the rational use of drugs in Beijing. Zhongguo Zhong Yao Za Zhi 2018;43:1049-53.

7. Qin D, Du PC, Wang AP, et al. Discussion on Chinese Medicinal Pharmacovigilance Based on the Literature Study on the Status of Pharmacovigilance. Proceedings of the 13th Academic Exchange Conference of Beijing Society of Environmental Mutagens in 2014 2014:220-4.
8. Yang YF. Discussion on clinical applied principle in guidance Ibr clinical application of Chinese patent medicine. The Chinese Journal of Clinical Pharmacology 2012,28:618-20.

9. Wang $\mathrm{SC}, \mathrm{Li} \mathrm{H}, \mathrm{Xu}$ L. Clinical guidelines for diagnosis and treatment of pediatric diseases in Chinese medicine: Allergic rhinitis in children. China Journal of Traditional Chinese Medicine and Pharmacy 2016,31:1352-5.

10. Chinese Pharmacopoeia Commission. Chinese Pharmacopeia. Beijing: Chemical Industry Press, 2005:69.

11. Li XL, Zhang YZ. The experimental study of anti-allergic effects of volatile oil from Flos Magnoliae. Chinese Journal of Hospital Pharmacy 2002,22:520-1.

12. Fan W, Fan L, Peng C, et al. Traditional Uses, Botany, Phytochemistry, Pharmacology, Pharmacokinetics and Toxicology of Xanthium strumarium L: A Review. Molecules 2019;24:359.

13. Dai Y, Bi PX, Chen YB. Inhibitory Effect of Xanthium sibiricum Fruits on Immediate Allergic Reactions. Chinese Wild Plant Resources 2002;21:61-4.

14. Zhang C, Wei P, Kou W, et al. Effect of Astragalus membranaceus on Peripheral Blood Th17 in Patients with Allergic Rhinitis. Zhongguo Zhong Xi Yi Jie He Za Zhi 2016;36:1364-8.

15. Song ZQ, Lin L, Zhu YF. The enhancement of Astragalus Polysaccharide on specific immunotherapy in asthmatic mouse. Chinese Journal of Immunology 2010;26:132-5.

16. Zhang M, Ren Y, Song CS, et al. Effect and mechanism of bishudiwan(BSDW) against allergy. Zhongguo Zhong Yao Za Zhi 2003;28:540-4.

17. Tong L, Liu JL, Wang JX, et al. Effect of Yupingfeng granule on cytokines of allergic rhinitis induced by OVA in rats. China Journal of Chinese Materia Medica 2016;41:728-30.

18. Fan XC, Niu CF. The Anti-inflammatory Activity of BiYuanTongQiao granule. Guiding Journal of Traditional Chinese Medicine and Pharmacy 2010;8:71-2.

19. Wang XH, Guo JF, Gu XZ. Effects of XinQin granule combined with fluticasone propionate on immunerelated factors and nasal function in patients with allergic rhinitis. Hebei Journal of Traditional Chinese Medicine 2018;40:1371-4.

20. Zhang M, Song CS, Ren Y, et al. Effects of Bishudiwan(BSDW) on $\mathrm{T}$ cell and related cytokines and $\mathrm{IgE}$ regulations in guinea pig model of allergic rhinitis. Journal of Beijing University of Traditional Chinese Medicine 2004,27:53-5.

21. Respiratory Group of Pediatrics Branch of Chinese 
Medical Association, Pediatrics Branch of Chinese Society of Traditional Chinese Medicine, Special Committee of Drug Clinical Evaluation and Research of China Association of Traditional Chinese Medicine, et al. Expert consensus on clinical application of Yupingfeng granules in children with respiratory diseases. Chin J Appl Clin Pediatr 2018;33:241-6.

22. Yang H. Clinical observation of Yupingfeng Granules in treating allergic rhinitis in children. Journal of Pediatrics of Traditional Chinese Medicine 2010;6(2):23-5.

23. Xu SF. Clinical observation of Yupingfeng granules combined with Loratadine syrup in treating children with allergic rhinitis. Contemporary Medical Symposium 2014;12(19):172-3.

24. Luo Q, Zhang CS, Yang L, et al. Potential effectiveness of Chinese herbal medicine Yu ping feng san for adult allergic rhinitis: a systematic review and meta-analysis of randomized controlled trials. BMC Complement Altern Med 2017;17:485.

Cite this article as: Liang J, Gu Q. Current status of Chinese herbal medicine to treat allergic rhinitis in children: from the perspective of Western medicine-a narrative review. Transl Pediatr 2021;10(12):3301-3308. doi: 10.21037/tp-21-553
25. Wang LQ, Yang Q, Cheng L. Meta-analysis of traditional Chinese medicine Yupingfeng granules in the treatment of allergic rhinitis. Chinese Journal of Otorhinolaryngology in Integrative Medicine 2018;26:223-30.

26. Chen MY, Wang JR, Wu RS. Experimental study on BiYuanTongQiao granule relieving allergic rhinitis by regulating SP-A level and Th1/Th2/Th17 cell balance. Chinese Journal of Immunology 2020;36:2846-9.

27. Chen ZW, Liu LQ, Gao Y, et al. The effect of BiYuanTongQiao granule on nasal mucociliary transport function and level of inflammatory factors in children with chronic sinusitis. Journal of Clinical Medicine in Practice 2016;20:83-6.

28. Hu CF, Chen X, Tu Y, et al. Effects of BiYuanTongQiao granule combined with lupatadine fumarate on trace elements and ventilation function in patients with allergic rhinitis. Chinese Journal of Otorhinolaryngology in Integrative Medicine 2021,29:46-9. 